\title{
OVCCA Web Application as Supplementary Material to Facilitate Health Literacy Regarding Carcinogenic Human Liver Fluke: A Randomized Controlled Trial in Thailand
}

\author{
Oranard Wattanawong ${ }^{1}$, Tiwakron Prachaiboon ${ }^{2,3,4}$, Thirayu Meererksom ${ }^{3}$, \\ Nathkapach Kaewpitoon Rattanapitoon ${ }^{3 *}$, Schawanya Kaewpitoon \\ Rattanapitoon ${ }^{3}$, Pannee Banchonhattakit ${ }^{4}$, Sarawut Boonsuk ${ }^{5}$, Thongroo \\ Kophachon ${ }^{1}$
}

\begin{abstract}
Background: Liver fluke, Opisthorchis viverrini, is associated to cholangiocarcinoma which is found frequently in some areas of Southeast Asian countries particularly in Thailand, Lao People Republic Democratic, Cambodia. This study sought to investigate the effects of an $O$. viverrini and cholangiocarcinoma (OVCCA) web application to facilitate health literacy regarding $O$. viverrini in Northeast Thailand. Methods: A randomized controlled trial study was performed among an intervention group $(\mathrm{n}=63)$ and a control group $(\mathrm{n}=63)$ during a one-year period from July 2019 to May 2020. The intervention group received the health literacy promotion program of $O$. viverrini information through the OVCCA web application for 6 weeks. The control group received an activity package from the public health department. The success of the program was evaluated at week 24 after the groups finished the last activity. ANCOVA, t-test and multiple logistic regression were used for data analysis for both groups. Results: The scores for knowledge; ability to access, understand, appraise, and apply information; motivation for protection; and practice of $O$. viverrini prevention were significantly higher for the intervention group than for the control group. The results indicated that a health literacy promotion program through an OVCCA web application could be advantageous for preventing and controlling $O$. viverrini infection. Conclusion: This intervention may be used as a potential strategy and guideline for self-care and health promotion in other endemic areas.
\end{abstract}

Keywords: Health literacy, Carcinogenic human liver fluke, Opisthorchis viverrini, OVCCA web application, Thailand

Asian Pac J Cancer Prev, 22 (9), 3045-3052

\section{Introduction}

Infection caused by the liver fluke, Opisthorchis viverrini, is a neglected tropical disease that affects health and poverty, particularly in Southeast Asian countries such as Thailand, Lao People's Democratic Republic, Cambodia, and the central part of Vietnam (Hotez et al., 2015). Nine million people per year suffer from $O$. viverrini infection (Sithithaworn et al., 2014). $O$. viverrini infection is associated with hepatobiliary diseases, including cholangiocarcinoma, mainly in northeastern Thailand and Laos because people in those areas extensively consume raw or half-cooked fish (Kaewpitoon et al., 2008; Chudthaisong et al., 2015; Saiyachak et al., 2016). In Thailand, more than 6 million people are at risk of liver fluke infection (Sithithaworn et al., 2012), which has been found to have the highest prevalence in the northeast $(16.6 \%)$ and north $(10 \%)$ (Wongsaroj et al., 2014). The infection is frequently found in men over 35 years of age and in agricultural occupations (Kaewpitoon et al., 2015). The infection results from the consumption of raw or undercooked freshwater cyprinoid fish. Many people dose with praziquantel as a treatment for an episode of the infection, and they still have a high risk for them of long-term consequences (Sithithaworn et al., 2012; Saengsawang et al., 2013). Effective prevention and control campaigns are needed to decrease the problem in the population at risk of disease. Health literacy (HL) represents the cognitive skills and social skills that determine the motivation and ability of

${ }^{1}$ Division of General Communicable Diseases, Department of Disease Control, Ministry of Public, Health, Nonthaburi, Thailand. ${ }^{2}$ Department of Public Health, Faculty of Public Health, Khon Kaen University, Khon Kaen, Thailand. ${ }^{3}$ Parasitic Disease Research Center, Suranaree University of Technology, Nakhon Ratchasima, Thailand. ${ }^{4}$ Faculty of Public Health, Valaya Alongkorn Rajabhat University, Thailand. ${ }^{5}$ Department of Health, Ministry of Public Health, Khon Kaen, Thailand. *For Correspondence: nathkapach.ratt@sut.ac.th 
individuals to promote and maintain good health (World Health Organization, 1998). People's competencies to access, understand, judge and apply health information in healthcare, disease prevention, and health promotion are based on their HL level and the factors associated with the specific population (Denuwara and Gunawardena, 2017). HL is a concept used in healthcare, disease prevention, and health promotion settings. It includes the following concepts: (1) access refers to the ability to seek, find and obtain health information; (2) understand refers to the ability to comprehend the health information that is accessed; (3) appraise describes the ability to interpret, filter, judge and evaluate the health information that is accessed; and (4) apply refers to the ability to communicate and use the information to make a decision to maintain and improve health (Sørensen et al., 2012). Therefore, HL is a good way to combat serious liver fluke infections in epidemic areas. Ferrucci et al., (2021) suggest that this approach provides a basis for future artificial intelligence-based approaches. We examined $\mathrm{HL}$ for $O$. viverrini and found that the population at risk of infection from this liver fluke had a high level of understanding and good practices after intervention (Prachaiboon et al., 2021). Recently, mobile and web applications for health care have increased worldwide, including in Thailand. The sustainability of such an application depends on the use and function of mobile and web applications (Akhbardeh et al., 2015; Anderson et al., 2016; Radovic et al., 2016). A mobile application screening test has been used to screen for $O$. viverrini infection, and this tool proved to be simple, rapid and low cost (Kaewpitoon $\mathrm{N}$ et al., 2018). Therefore, this study aimed to investigate the effects of health literacy promotion programs and an OVCCA web application on the prevention of $O$. viverrini infection in Northeast Thailand.

\section{Materials and Methods}

\section{Study design}

This randomized controlled trial was conducted in Roi Et Province, Northeast Thailand, for one year between July 2019 and May 2020 (Figure 1). A total population of 126 individuals living in Phon Tong District, with those in Kham Na Di Subdistrict as the intervention group and those in Sawang Subdistrict as the control group. The participants were selected through randomized allocation and completed self-administered questionnaires (Figure 2).

\section{Health literacy promotion program through OVCCA web application}

A health literacy promotion program (HLPP) and $O$. viverrini and cholangiocarcinoma (OVCCA) web application on $O$. viverrini prevention were performed among the intervention and control groups in Northeast Thailand. The intervention group received the HLPP of $O$. viverrini information through the OVCCA web application, while the control group received general information on $O$. viverrini from local health personnel as an annual campaign. The intervention group joined the 6-week HLPP through the OVCCA web application, which was developed as an open-source web application by the Department of Disease Control, Ministry of Public Health, which is affiliated with Parasitic Disease Research Center, Nakhon Ratchasima, Thailand. The application could be accessed by smartphone, tablet or computer. The HLPP program included 14 activities: week 1: (A1) Community participatory action with relationship building and (A2) Collecting data before intervention; self-checking using screening test, learning $O$. viverrini knowledge by (A3) animation of life cycle, signs and symptoms, prevention and control; (A4) VDO clip of pathogenesis and pathology, signs and symptoms, diagnosis, treatment, prevention and control; (A5) infographic images of morphology, life cycle, pathogenesis and pathology, diagnosis, prevention and control; (A6) pictures of morphology, life cycle, and diagnosis; (A7) health behavior daily record; week 2: A2-A7; (A8) lecture clip of $O$. viverrini knowledge; (A9) case-based learning using a VDO clip story of $O$. viverrini patients; week 3: A2-9; (A10) sharing experiences by role model by people who have succeeded in liver fluke prevention; week 4: A2-7; (A11) safety cooking demonstration using a VDO clip; week 5: A2-11; (A12) local folk songs (Esan song) clip related to liver fluke prevention; week 6: A2-12; (A13) home visit intervention group by health village volunteers, local health officers, and researchers; and (A14) meeting, reflecting, and returning data to communities and individuals so the supplemented intervention group could continue to receive health education.

\section{Assessment of health literacy promotion program through OVCCA web application}

A comprehensive health literacy questionnaire from the European Health Literacy Survey (HLS-EU-Q47) was applied and assessed all participants at week 24 . This questionnaire was grounded in a conceptual framework and operationalized through a matrix with 7 dimensions, including four information-processing domains (finding, understanding, judging, and applying) and three health domains (health care, disease prevention, and health promotion) (Kaewpitoon et al., 2018). It consisted of 4 parts: 1) demographic characteristics; 2) knowledge about O. viverrini; 3) 4 HL skills: access information, understand information, appraise information, and apply information; and 4) practice of $O$. viverrini prevention and control. The questionnaire was validated by 7 experts and tested for reliability. The Kuder-Richardson 20 coefficient of knowledge about $O$. viverrini was 0.74 . The Cronbach's alpha coefficients of $\mathrm{HL}$ and $O$. viverrini infection practices were 0.87 and 0.76 , respectively. Knowledge about $O$. viverrini infection was calculated and analyzed at three levels. The section on knowledge about $O$. viverrini consisted of 10 questions, with a correct answer $=1$ and an incorrect answer $=0$, and the scores were interpreted as high at $80 \%$ (8-10 points), moderate at $60 \%-79 \%$ (6-8 points), and low at $0-59 \%$ (0-5 points). The HL section included 47 questions that were interpreted as $0-25$ points for inadequate, $>25$ to 33 points for problematic, $>33$ to 42 points for sufficient, and $>42$ to 50 points for excellent. The section on $O$. viverrini prevention and control practice 
had 10 questions that were interpreted as practice at $100 \%$ (10 points) and nonpractice at less than $100 \%(0-9$ points). In this study, raw cyprinoid fish consumption was dichotomized (yes/no).

\section{Statistical analysis}

All analyses were performed using Stata version 10.0 (Stata Corp., College Station, TX). ANCOVA, t-test and multiple logistic regression were used to analyze raw cyprinoid fish consumption and HL scores by crude adjusted mean difference, adjusted relative ratios (ORadj) and $95 \%$ confidence interval (CI). All test statistics were two-sided, and a p-value of less than 0.05 was deemed statistically significant.

\section{Ethical Considerations}

This study was approved by the Ethics Committee in Human Research of Nakhon Ratchasima Health Provincial Office, Thailand 2018 (Reference no. NRPH041).

\section{Results}

The majority of the intervention group were female (66.6\%), aged 26-35 years $(49.2 \%)$, with a high school education $(54.0 \%)$, working in agriculture (famer) (52.4\%), and with an income of 36-160 US dollar (42.9\%). The majority of the control group were female (69.8\%), aged $26-35$ years $(47.6 \%)$, with a high school education (57.1\%), working in agriculture (famer) $(49.2 \%)$, and with an income of 36-160 US dollar (41.3\%) (Table 2). Thus, there were no differences in demographic data between the intervention and control groups. The results reveal that after the 6-week intervention and then at the 24-week follow-up, the mean scores of knowledge ( $p$-value $=0.001$ ), ability to access information ( $p$-value $=0.018$ ), ability to understand information $(\mathrm{p}$-value $=0.002)$, ability to appraise information ( $\mathrm{p}$-value $=0.038$ ), ability to apply information ( $\mathrm{p}$-value $=0.043$ ), and motivation for protection $(\mathrm{p}$-value $=0.005)$ of $O$. viverrini prevention and control were higher for the intervention group than for the control group, and the difference was statistically significant (Table 1). In addition, the adjusted odds ratios (ORadj) of raw fish consumption for the health literacy promotion program and OVCCA web application for the prevention of $O$. viverrini infection were analyzed for both the intervention group and the control group, and the results indicated that after the intervention, the mean scores of practice for $O$. viverrini prevention were higher for the intervention group than for the control group $($ ORadj $=2.91: 95 \%$ CI: 1.11-7.67; $\mathrm{p}$-value $=0.030)$, and the difference was statistically significant (Table 3 ).

\section{Discussion}

Infection by the liver fluke, $O$. viverrini, is associated with hepatobiliary diseases, including cholangiocarcinoma (Kaewpitoon et al., 2008; Chudthaisong et al., 2015; Saiyachak et al., 2016), and more than 9 million people per year suffer $O$. viverrini infection in Thailand (Sithithaworn et al., 2012). The infection results from the consumption of raw or undercooked freshwater cyprinoid fish, and many people suffer long-term consequences (Saengsawang et al., 2013). Prevention and control campaigns are needed to decrease the problem. HL represents cognitive skills and social skills related to people's competency to access, understand, judge and apply health information in healthcare, disease prevention, and health promotion (Denuwara and Gunawardena, 2017; Duong et al., 2017). Recently, innovative technologies, including web-based, mobile and web applications, have increasingly been used as a medium for gathering and exchanging health information. Wolpin et al., (2010) developed an open-source web application that measures user reaction times, subjective quality ratings, and accuracy in completing tasks across different audio files created by text-to-speech engines. This study was successful in building and piloting the web application for a health intervention to improve users' knowledge and health practices. In addition, mobile and web applications have been used to increase health screening tests for $O$. viverrini infection. The study showed that compared

Table 1. Background Variables in Intervention and Control Groups

\begin{tabular}{|c|c|c|}
\hline Variables & $\begin{array}{l}\text { Intervention } \\
\text { group n }(\%)\end{array}$ & $\begin{array}{c}\text { Control group } \\
\mathrm{n}(\%)\end{array}$ \\
\hline \multicolumn{3}{|l|}{ Gender } \\
\hline Male & $21(33.4)$ & $19(30.2)$ \\
\hline Female & $42(66.6)$ & $44(69.8)$ \\
\hline \multicolumn{3}{|l|}{ Age group (years) } \\
\hline $18-25$ & $2(3.2)$ & $3(4.8)$ \\
\hline $26-35$ & $31(49.2)$ & $30(47.6)$ \\
\hline $36-45$ & $15(23.8)$ & $16(25.4)$ \\
\hline $46-55$ & $10(15.8)$ & $7(11.1)$ \\
\hline $56-65$ & $2(3.2)$ & $5(7.9)$ \\
\hline$>65$ & $3(4.8)$ & $2(3.2)$ \\
\hline \multicolumn{3}{|l|}{ Education } \\
\hline Illiterate Primary & $21(33.3)$ & $17(27.0)$ \\
\hline Secondary & $34(54.0)$ & $36(57.1)$ \\
\hline Academic & $8(12.7)$ & $10(15.9)$ \\
\hline \multicolumn{3}{|l|}{ Job status } \\
\hline Housewife & $12(19.0)$ & $14(22.2)$ \\
\hline Employed & $6(9.5)$ & $5(7.9)$ \\
\hline Famer & $33(52.4)$ & $31(49.2)$ \\
\hline Government officer & $5(7.9)$ & $8(12.7)$ \\
\hline Trade & $7(11.0)$ & $5(7.9)$ \\
\hline \multicolumn{3}{|c|}{ Family income (\$, per month) } \\
\hline$<35$ & $7(11.1)$ & $6(9.5)$ \\
\hline $36-160$ & $27(42.9)$ & $26(41.3)$ \\
\hline $161-350$ & $15(23.8)$ & $17(27.0)$ \\
\hline $351-500$ & $9(14.3)$ & $7(11.1)$ \\
\hline $501-650$ & $5(7.9)$ & $7(11.1)$ \\
\hline \multicolumn{3}{|c|}{ Raw or undercooked fish consumption } \\
\hline Yes & $58(92.1)$ & $57(90.5)$ \\
\hline No & $5(7.9)$ & $6(9.5)$ \\
\hline
\end{tabular}

Data presented as frequency (\%).

Asian Pacific Journal of Cancer Prevention, Vol $22 \mathbf{3 0 4 7}$ 
Table 2. Results of Mean \pm Standard Deviation and Adjusted Mean Difference, with 95\% Confidence Intervals Between Intervention Group and Control Group among Health

\begin{tabular}{|c|c|c|c|c|c|c|c|c|}
\hline \multirow[t]{2}{*}{ Variables } & \multirow[t]{2}{*}{ Duration } & \multicolumn{2}{|c|}{ Intervention group } & \multicolumn{2}{|c|}{ Control group } & \multirow{2}{*}{$\begin{array}{l}\text { Adj. mean } \\
\text { difference }\end{array}$} & \multirow[t]{2}{*}{$95 \% \mathrm{CI}$} & \multirow[t]{2}{*}{ P-value } \\
\hline & & Mean & SD & Mean & SD & & & \\
\hline \multirow[t]{2}{*}{ Knowledge } & Baseline & 8.3 & 0.28 & 8.6 & 0.14 & & & \\
\hline & Follow & 9.1 & 0.16 & 8.6 & 0.14 & 0.64 & $0.26-1.02$ & 0.001 \\
\hline \multirow[t]{2}{*}{ Ability to access information } & Baseline & 18.5 & 1.1 & 17.3 & 1.38 & & & \\
\hline & Follow & 22.7 & 1.25 & 17.9 & 1.4 & 4.55 & $0.80-8.30$ & 0.018 \\
\hline \multirow[t]{2}{*}{ Ability to understand information } & Baseline & 26.4 & 1.12 & 26.8 & 1.23 & & & \\
\hline & Follow & 29.8 & 1.23 & 25.1 & 1.25 & 5.49 & $2.11-8.87$ & 0.002 \\
\hline \multirow[t]{2}{*}{ Ability to appraise information } & Baseline & 24.4 & 0.84 & 25.7 & 0.97 & & & \\
\hline & Follow & 28.9 & 1.29 & 26.6 & 0.96 & 3.01 & $0.16-5.86$ & 0.038 \\
\hline \multirow[t]{2}{*}{ Ability to apply information } & Baseline & 30.6 & 1.28 & 30.2 & 1.52 & & & \\
\hline & Follow & 34.6 & 1.42 & 32.1 & 1.43 & 2.26 & $0.51-6.04$ & 0.043 \\
\hline \multirow[t]{2}{*}{ Protection motivation theory } & Baseline & 73.6 & 1.24 & 71.6 & 1.24 & & & \\
\hline & Follow & 77.3 & 1.47 & 71.3 & 1.23 & 4.89 & $1.52-8.26$ & 0.005 \\
\hline
\end{tabular}

to laboratory tests, these tools are simple, rapid and low cost (Kaewpitoon et al., 2018). Internet-based health technology, including web-based resources, patient portals, and mobile phone apps, is increasingly being used to help manage chronic disease, educate people, and monitor users' condition (Madrigal and Escoffery, 2019). The participants have been highly satisfied and use such technology frequently. Khademian et al (2020) assessed web-based health information seeking and eHealth literacy among Iranian college students and showed that participants usually searched for illnesses, symptoms, and treatments after they became sick and paid little attention to other aspects related to integral health. Prachaiboon et al (2021) conducted a cross-sectional analytical study of HL among 1,163 respondents from Northeast Thailand and found that they had inadequate access to health information, inadequate understanding of health information, inadequate appraisal of health information and inadequate health information associated with raw cyprinoid fish consumption. This study indicated an urgent need to improve $O$. viverrini information, particularly in HL, and change people's eating behaviors, traditions, and contexts, including feeding dogs and cats. Therefore, the effects of health literacy promotion

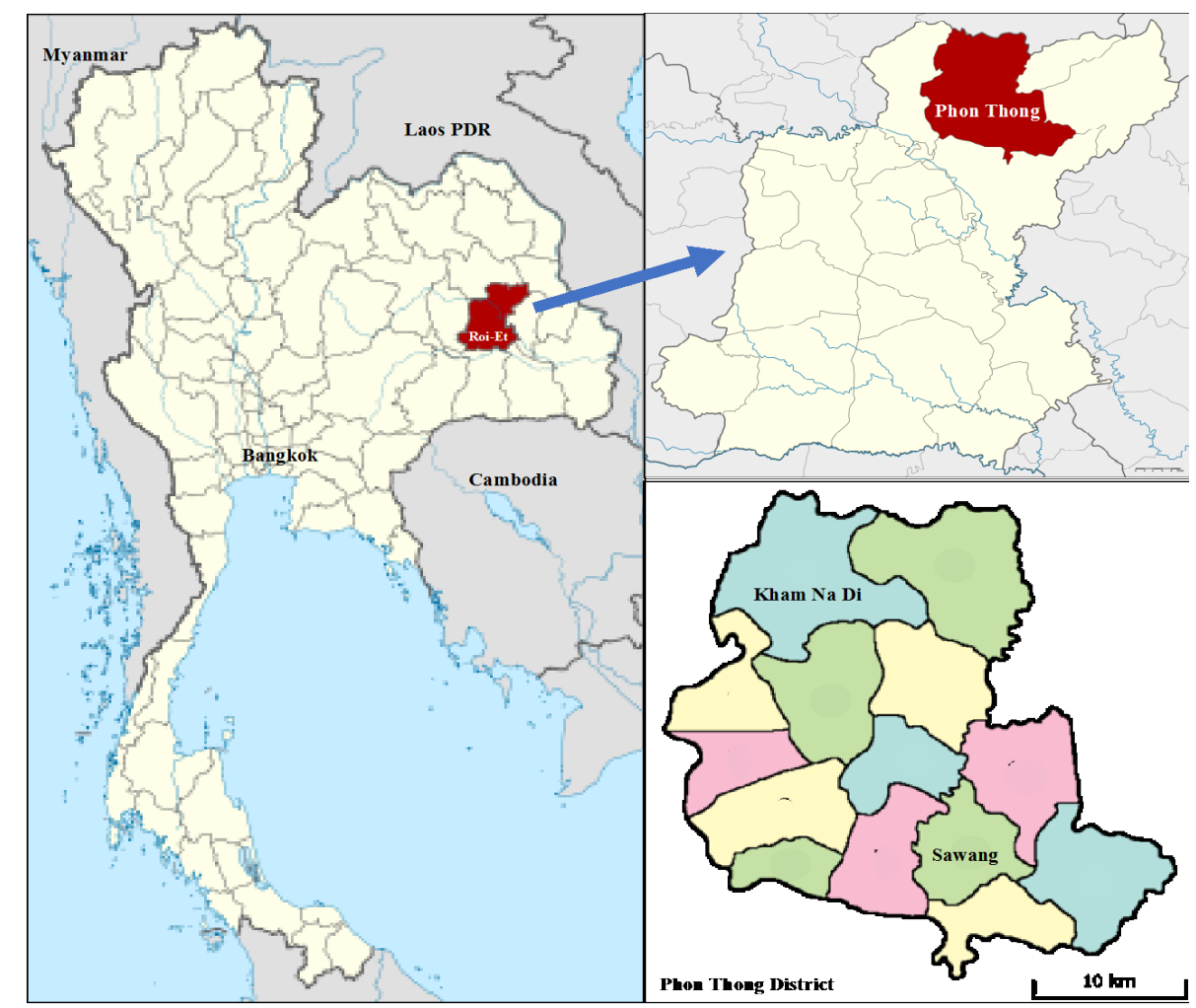

Figure 1. The Study Area, including the Kham Na Di and Sawang Subdistricts, where the Populations were Randomized into Intervention and Control Groups. 
OVCCA Web Application as Supplementary Material to Facilitate Health Literacy Regarding Carcinogenic HLF

Table 3. Odds Ratios (ORadj) of Raw Fish Consumption and 95\% Confidence Intervals for Health Literacy Promotion Program and OVCCA Web Application for the Prevention of $O$. viverrini Infection Between the Intervention Group and Control Group Using Multiple Logistic Regression

\begin{tabular}{|c|c|c|c|c|c|c|c|c|}
\hline \multirow[t]{2}{*}{ Prevention of O. viverrini infection } & \multicolumn{2}{|c|}{ Intervention group } & \multicolumn{2}{|c|}{ Control group } & \multirow[t]{2}{*}{ Crude OR } & \multirow[t]{2}{*}{ Adj. OR } & \multirow[t]{2}{*}{$95 \% \mathrm{CI}$} & \multirow[t]{2}{*}{ P-value } \\
\hline & $\mathrm{n}$ & $\%$ & $\mathrm{n}$ & $\%$ & & & & \\
\hline \multicolumn{9}{|l|}{ Baseline } \\
\hline nonpractice & 58 & 92.1 & 57 & 90.5 & 1 & 1 & 1 & \\
\hline practice & 5 & 7.9 & 6 & 9.5 & 0.81 & 0.89 & $0.23-3.33$ & 0.864 \\
\hline \multicolumn{9}{|l|}{ Follow } \\
\hline nonpractice & 45 & 71.4 & 55 & 87.3 & 1 & 1 & 1 & \\
\hline practice & 18 & 28.6 & 8 & 12.7 & 2.75 & 2.91 & $1.11-7.67$ & 0.03 \\
\hline
\end{tabular}

programs and OVCCA web applications on $O$. viverrini prevention were examined in this study. The intervention group was assigned to receive information on $O$. viverrini through HL and an OVCCA web application, while the control group received general information on $O$. viverrini from local health personnel as an annual campaign. The results reveal that the intervention can increased the participants' knowledge, ability to access information, ability to understand information, ability to appraise information, ability to apply information and motivation for protection from $O$. viverrini infection. These results were similar to those of previous studies about other diseases; in particular, Puttapunyo et al., (2016) reported the effects of health behavioral development programs on the HL and weight-loss behavior of overweight personnel at Roi Et Hospital, Mueang District, Roi Et Province, Thailand. The study revealed that after the experiment, the participants had significantly higher HL and weight loss behavior than before the experiment, and the difference was statistically significant. Furthermore, Padchasuwan et al., (2018) conducted a study of secondary school students in Northeast Thailand and suggested that HL can be a desirable strategy for informing those with a lower practice level regarding liver fluke prevention and control. Continuous and extensive health education programs help people obtain more information on diseases and increase their HL.

Our study found that the participants in the intervention group had increased HL regarding $O$. viverrini prevention and control after they received information on

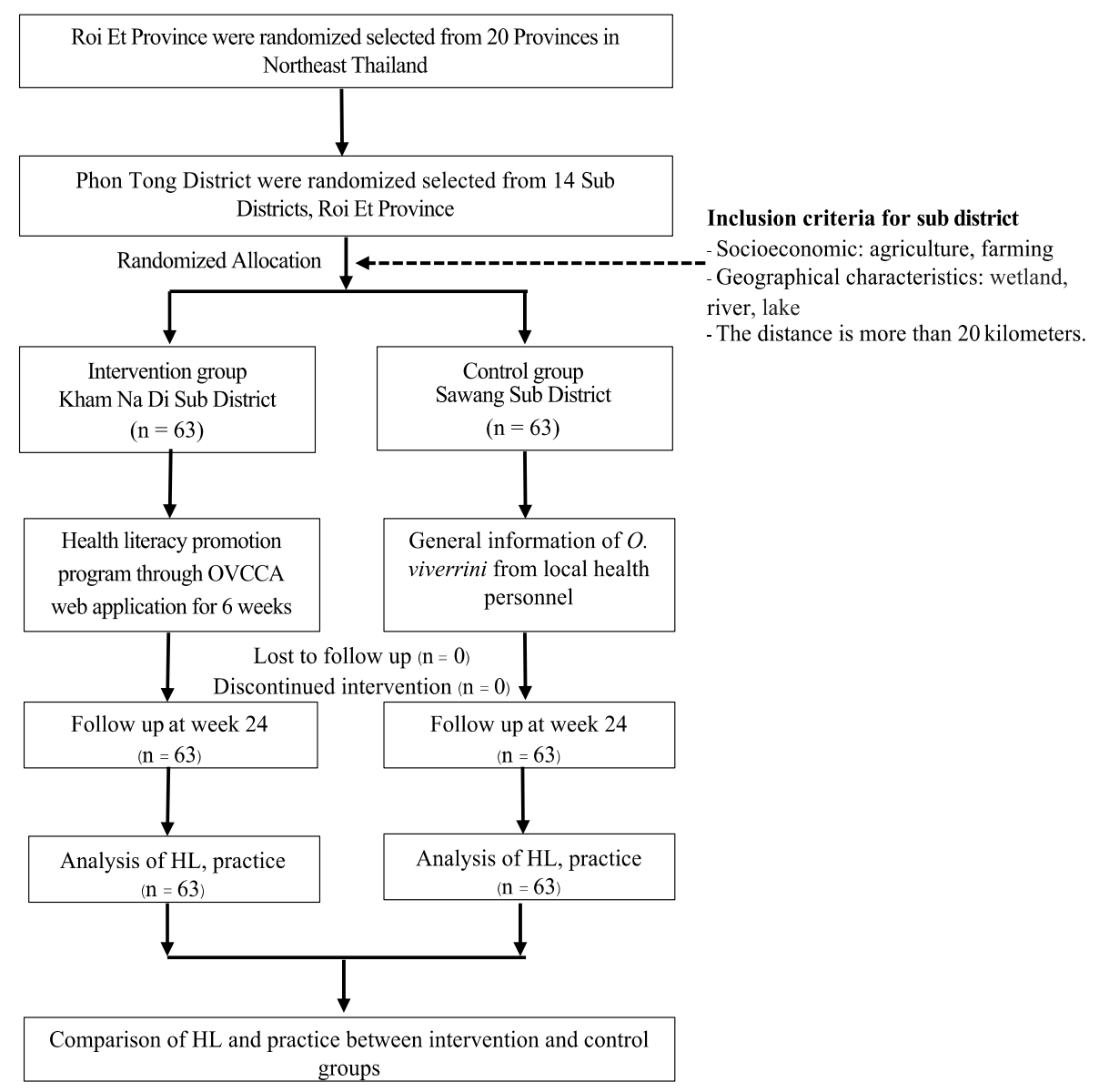

Figure 2. CONSORT Diagram of This Study 


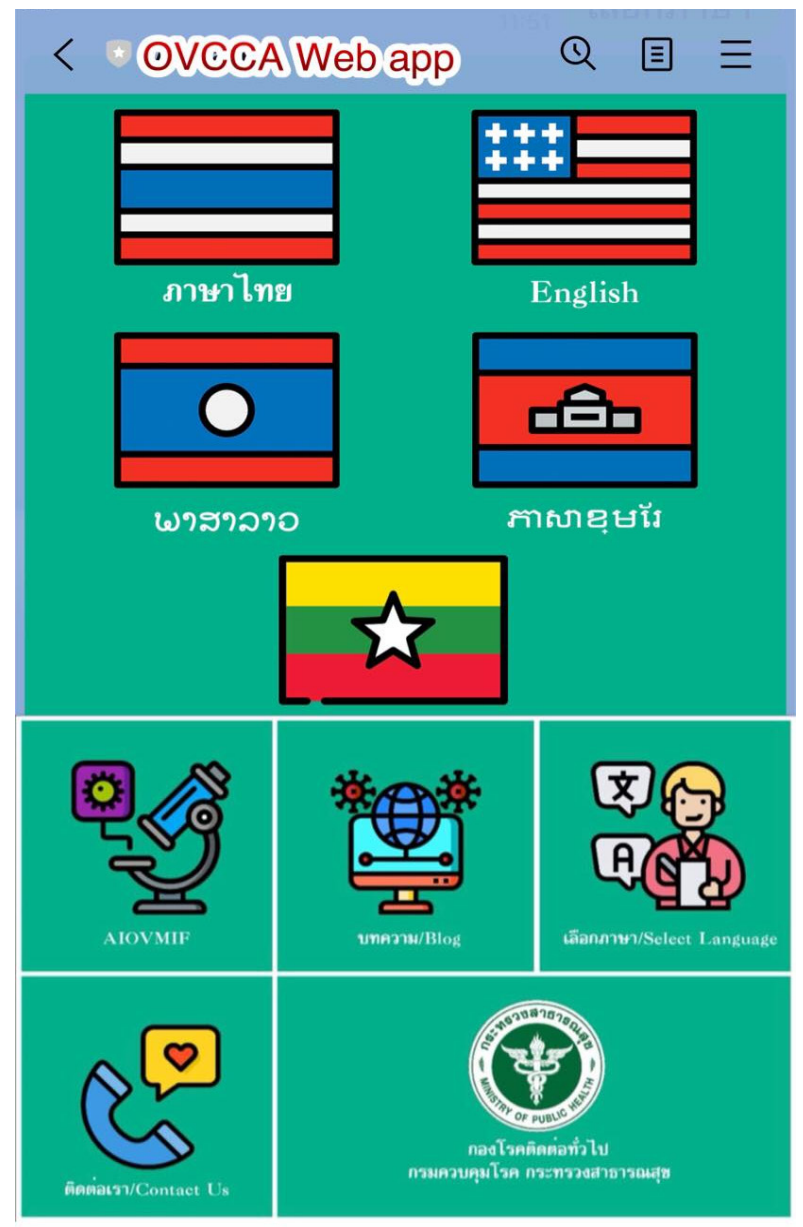

圆

Menu $\cdot$

Figure 3. OVCCA Web Application; an Open-Source Web Application was Developed by the Department of Disease Control, Ministry of Public Health, which is Affiliated with the Parasitic Disease Research Center, Nakhon Ratchasima, Thailand

O. viverrini during a 6-week health behavioral development program with 15 activities through the HL program and OVCCA web application. This result is similar to that of Boom (1971), who suggested that a successful behavioral modification program should be composed of a variety of activities. Our program included lectures using PowerPoint, posters, brochures, pamphlets, liver fluke slides under a microscope, VDO clips, daily health behavior records, idea sharing, safety cooking demonstrations, and local folk songs. The program was advantageous in helping the participants understand more information and motivating them to prevent $O$. viverrini infection. In addition, the activities, including discussion of $O$. viverrini information and idea sharing by role models who had succeeded in liver fluke prevention and control, supported behavioral change and HL in this group. These results were similar to those of other studies on motivation for behavioral change. Iftikhal (2018) applied protection motivation theory in terms of disease prevention behaviors for liver fluke infection in Ban Nong Hoi grade school, Muang District, Sakon Nakhon Province, Thailand, by using variations on 4 activities. The study found that the group that received the program had higher mean scores for knowledge, perceived severity, perceived susceptibility, response efficacy, efficacy expectation, health promotion, and disease prevention behaviors for liver fluke than the control group at the level of significance. Panithanang et al., (2018) indicated that the experimental group that received a health behavioral modification program based on health education, self-efficacy, motivation, social support and networking had significantly higher scores than those in the comparison group. Participants gained the correct knowledge and had higher self-efficacy, expectations, and practices regarding liver fluke prevention. The educational intervention consisted of seven training sessions (introduction to cholangiocarcinoma, risk factors, complications, benefits and barriers to proper consumption of cooked fish, carcinogenic agents, behavioral protection, and self-efficacy in applying preventive behaviors), and the participants showed a significant increase in knowledge, perceived susceptibility, perceived severity, perceived benefits, perceived barriers, cues to action, self-efficacy, and cholangiocarcinoma preventive behaviors (Srithongklang et al., 2019). In our study, the intervention group received motivation and continued support for obtaining information and $O$. viverrini prevention through the OVCCA web application.

The HL program and OVCCA web application were advantageous in enabling the participants to access information and manage their health. The experimental group had better $O$. viverrini infection prevention practices than the control group. In the OVCCA web application, the contents comprised images, animations, clips, screening tests, and local folk songs that benefited users by improving their knowledge and management of their health. The varieties of content and materials that have proven to be advantageous have increasingly been technology based, mainly animations, clips and songs. An interactive animation program for improving the knowledge of students studying liver fluke showed that the students achieved a significantly improved perception. The use of the animated program facilitated education about liver fluke and was good supplementary learning material for the students, particularly when they were learning about serious concepts (Bukkhunthod et al., 2020). The success of our web application is similar to that of Wang et al., (2015), who successfully designed a recommendationbased mobile web application to assist patients in efficiently seeking online health information at any time, anywhere and via any devices using a collaborative filtering approach to recommend health information. CarrascoHernandez et al., (2020) reported the long-term efficacy of a mobile app supporting psychopharmacological therapy for smoking cessation. They also performed a complementary assessment of the applied innovative technology and found that the proposed mobile health solution complementing psychopharmacological therapy showed greater efficacy in achieving 1-year tobacco abstinence than psychopharmacological therapy alone. This result suggests that such technology provides a basis for future artificial intelligence-based approaches. In addition, Ferrucci et al., (2021) reported that a specific web-based mobile app provided adequate and personalized support to complex health care populations. The results 
showed good family and community engagement and revealed some limitations that need to be addressed. Patients described themselves as satisfied or very satisfied. Health care providers reported improved communication with colleagues and the need to support data quality. With increased access to the internet and technology, there are many opportunities for utilizing the internet, electronic health applications, and information for the prevention and management of diseases. Our results show that an OVCCA web application is beneficial for promoting health and disease prevention, particularly HL in terms of $O$. viverrini.

In conclusion, this study showed that the health literacy promotion program and OVCCA web application for the prevention of $O$. viverrini infection could serve as a potential strategy and guideline for self-care and health promotion for the prevention of $O$. viverrini infection in Northeast Thailand. In addition, it indicated that the program and OVCCA web application could enable users to access information, understand information, make decisions and appraise information, apply information and practice prevention of $O$. viverrini infection.

\section{Author Contribution Statement}

Study conception and design: OW, TP, NKR, PB; data collection: TP, TM, SB; analysis and interpretation of results: OW, TP, NKR, SWR, PB, TK; draft manuscript preparation: OW, TP, NKR, SWR, PB; All authors reviewed the results and approved the final version of the manuscript.

\section{Acknowledgements}

The authors would like to thank the village health volunteers, village leaders, local health personals, sub-district administrative organization in the study areas for great assistant and collaboration.

\section{Funding Statement}

This research was supported by a Parasitic Disease Research Center under the grant of Suranaree University of Technology research and development fund, Suranaree University of Technology, Thailand 2019, and the Department of Disease Control, Ministry of Public Health, Thailand 2019.

\section{Conflicts of interest}

There are no conflicts of interest.

\section{References}

Akhbardeh F, Vasefi F, Tavakolian K, et al (2015). Toward development of mobile application for hand arthritis screening. Annu Int Conf IEEE Eng Med Biol Soc, 2015, 7075-8.

Anderson K, Burford O, Emmerton L (2016). App chronic disease checklist: protocol to evaluate mobile apps for chronic disease self-management. JMIR Res Protoc, 5, e204.

Bloom BS (1971). Handbook on formative and summative of student learning. New York: Mc Graw-Hill Book Company, pp 1-12.
Bukkhunthod P, Meererksom T, Pechdee P, et al (2020). Animation as supplementary learning material about carcinogenic liver fluke in classes for primary schoolchildren. $J$ Cancer Educ, 35, 14-21.

Carrasco-Hernandez L, Jódar-Sánchez F, Núñez-Benjumea F, et al (2020). A mobile health solution complementing psychopharmacology-supported smoking cessation: randomized controlled trial. JMIR Mhealth Uhealth, 8 , e17530.

Chudthaisong N, Promthet S, Bradshaw P (2015). Risk factors for Opisthorchis viverrini infection in Nong Khai Province, Thailand. Asian Pac J Cancer Prev, 16, 4593-96.

Denuwara HMBH, Gunawardena NS (2017). Level of health literacy and factors associated with it among school teachers in an education zone in Colombo, Sri Lanka. BMC Public Health, 17, 631.

Duong TV, Aringazina A, Baisunova G, et al (2017). Measuring health literacy in Asia: Validation of the HLS-EU-Q47 survey tool in six Asian countries. J Epidemiol, 27, 80-6.

Ferrucci F, Jorio M, Marci S, et al (2021). A web-based application for complex health care populations: usercentered design approach. JMIR Hum Factors, 8, e18587.

Hotez PJ, Bottazzi ME, Strych U, et al (2015). Neglected tropical diseases among the Association of Southeast Asian Nations (ASEAN): overview and update. PLoS Negl Trop Dis, 9, e0003575.

Iftikhal A (2018). The effects of applying protection motivation theory in term of disease prevention behaviors liver fluke in grade Ban Nong Hoi school, Muang district, Sakon Nakhon province. J Off DPC7 KK, 24, 75-87.

Kaewpitoon N, Kaewpitoon SJ, Meererksom T, et al (2018). Detection of risk groups for carcinogenic liver fluke infection by verbal screening questionnaire using a mobile application. Asian Pac J Cancer Prev, 19, 2013-19.

Kaewpitoon N, Kaewpitoon SJ, Pengsaa P, et al (2018). Opisthorchis viverrini: the carcinogenic human liver fluke. World J Gastroenterol, 14, 666-74.

Kaewpitoon N, Kootanavanichpong N, Kompor P, et al (2015). Review and current status of Opisthorchis viverrini infection at the community level in Thailand. Asian Pac J Cancer Prev, 16, 6825-30.

Khademian F, Roozrokh M, Aslani A (2020). Web-based health information seeking and eHealth literacy among college students. a self-report study. Invest Educ Enferm, 38, e08

Madrigal L, Escoffery C (2019). Electronic health behaviors among US adults with chronic disease: cross-sectional survey. J Med Internet Res, 21, e11240.

Padchasuwan N, Banchonhattakit P, Kaewpitoon N (2018). Health literacy associated with liver fluke prevention and control among secondary school students in Northeast Thailand. Suranaree J Sci Technol, 25, 307-18.

Panithanang B, Srithongklang W, Kompor P, et al (2018). The effect of health behavior modification program for liver fluke prevention among the risk group in rural communities, Thailand. Asian Pac J Cancer Prev, 19, 2673-80.

Prachaiboon T, Banchonhattakitc P, Rattanapitoon NK, et al (2021). Health literacy associated with raw cyprinoid fish consumption in Northeastern Thailand. Med Leg Up, 21, 1149-54.

Puttapunyo S, Toontom N, Putthanachote N (2016). Effectiveness of a health behavior development program on health literacy, weight loss behavior and weight of overweight personnel in Roi Et hospital, Mueang District, Roi Et Province, Thailand. J Nur Educ, 9, 42-59.

Radovic A, Vona PL, Santostefano AM, et al (2016). Smartphone applications for mental health. Cyberpsychol Behav Soc Netw, 19, 465-70. 
Saengsawang P, Promthet S, Bradshaw P (2013) Infection with Opisthorchis viverrini and use of praziquantel among a working-age population in northeast Thailand. Asian Pac J Cancer Prev, 14, 2963-6.

Saiyachak K, Tongsotsang S, Saenrueang T, et al (2016). Prevalence and factors associated with Opisthorchis viverrini nfection in Khammouane Province, Lao PDR. Asian Pac J Cancer Prev, 17, 1589-93.

Sithithaworn P, Andrews R, Shekhovtsov SV, et a. (2014). Opisthorchis viverrini and Opisthorchis felineus. Encyclopedia Food Safety, 2, 170-8.

Sithithaworn P, Andrews RH, Nguyen VD, et al (2012). The current status of opisthorchiasis and clonorchiasis in the Mekong Basin. Parasitol Int, 61, 10-6.

Sørensen K, Van den Broucke S, Fullam J, et al (2012). Health literacy and public health: a systematic review and integration of definitions and models. BMC Public Health, 12, 80 .

Srithongklang W, Panithanang B, Kompor P, et al (2019). Effect of educational intervention based on the health belief model and self-efficacy in promoting preventive behaviors in a cholangiocarcinoma screening group. J Cancer Educ, 34, 1173-80.

Wang SL, Kuo MH, Shiu YS, et al (2015). A recommendationbased mobile web application for health information service. Stud Health Technol Inform, 208, 337-41.

Wolpin S, Berry DL, Kurth A, et al (2010). Improving health literacy: a web application for evaluating text-to-speech engines. Comput Inform Nurs, 28, 198-204.

Wongsaroj T, Nithikathkul C, Rojkitikul W, et al (2014). National survey of helminthiasis in Thailand. Asian Biomed, 8 , 779-83.

World Health Organization (1998). Health promotion Glossar. Geneva: WHO Publications. Retrieved from https://www. who.int/healthpromotion/about/HPR\%20Glossary\%20 1998.pdf.

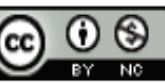

This work is licensed under a Creative Commons AttributionNon Commercial 4.0 International License. 